\begin{tabular}{|c|c|c|c|c|}
\hline Revista Praxis & ISSN: $1657-4915$ & Vol. 12 & $126-134$ & Enero - Diciembre de 2016 \\
\hline
\end{tabular}

\title{
TRANSFORMANDO LA UNIVERSIDAD EN COLOMBIA: HACIA LA CONSTRUCCIÓN DE NUEVOS ESCENARIOS
}

\author{
REMAKING THE UNIVERSITY IN COLOMBIA: TO THE BUILDING OF NEW STAGES
}

\author{
Lilia Cristina Romero-Hurtado', Doris Magaly Colina-de-Andrade²
}

\begin{abstract}
RESUMEN
Este articulo plantea la necesidad de transformar la universidad en Colombia, entendiendo que el desafío está en que las universidades se involucren en la vida social, apuesten por la transformación de educadores y de educandos en la construcción de un nuevo escenario educativo que consolide una sociedad justa e igualitaria, respondiendo a una visión integradora, misma que permita abordar problemas fuera de barreras disciplinarias y esquemas pedagógicos tradicionales, desarrolle competencias, conocimientos, habilidades que impacten y propicien ambientes abiertos y flexibles de educación. La universidad que debemos construir, haciendo eco de Ribeiro, es "una Utopía en el mundo de las ideas", aquella capaz de actuar como agencia de aceleración evolutiva de la sociedad, que sea vínculo o expresión moderna de la conciencia nacional, e interprete la realidad del País. Una universidad que forme a las nuevas generaciones para que afronten y asuman el futuro de manera responsable de cara a los compromisos de la nueva sociedad que se gesta, orientando sus esfuerzos a la investigación, pero por sobre todas las cosas, a la humanización de los saberes.
\end{abstract}

Palabras clave: Universidad, Transformación, sociedad, utopía, humanización de los saberes.

\begin{abstract}
This article raises the need to transform universities in Colombia, understanding that the challenge is to involve universities into the social life, betting on the transformation of the educators and the learners in the construction of a new eduactional setting, which consolidates a more fair and equal society. Responding to an integrative vision the same that allows dealing with problems outside disciplinary barriers and traditional pedagogic schemes, developing competences, knowledge and abilities that impact and propitiate open environments, flexibles of education. The university we must construct echoing Ribeiro is "An utopia in the world of the ideas", capable of acting as an acceleration of the evolutive agency of society, link or modern expression of the national conscience and that interpretates the reality of the country. A university that shapes the
\end{abstract}

Fecha de recepción: Octubre 06 de 2015 / Fecha de aceptación: Julio 27 de 2016

Tipología: Artículo de Reflexión

Para citar este artículo: Romero, H. L., \& Colina, A. D. (2016). Transformando la universidad en Colombia: hacia la construcción de nuevos escenarios. Praxis. Vol. 12, 126 - 134

1. Candidata a Doctora en Ciencias Políticas, Universidad Rafael Belloso Chacín. Especialista en Derecho Público y Magistra en Estudios Politico-Economicos. Abogada. Colombia. Email: gabiemerson@hotmail.com

2. Doctora en Ciencias Políticas. Magíster en Ciencias de la Educación, mención Gerencia educativa. Abogada. Miembro del Comité Académico del Doctorado en Ciencias Políticas. Universidad Privada Dr. Rafael Belloso Chacín, URBE. Maracaibo- Venezuela. Dra. Universidad Privada Dr. Rafael Belloso Chacín, URBE. Venezuela. Email: dorisdeandrade@gmail.com 
new generations for facing and assuming the future in a responsible way in the face of the compromises of the new society that is developing, gearing their efforts to the investigation, but overall to the humanization of the knowledge.

Keywords: University, transformation, society, utopia, humanization of the knowledge.

\section{INTRODUCCIÓN}

$\mathrm{R}$ eferirnos a la universidad, una de las instituciones más importantes dentro de la sociedad humana, no es tarea fácil, quienes han transitado por los campos de la formación sociohumanística en su ámbito, han sido testigos de los cambios, que ha sufrido, de hecho, el debate en torno a la educación en América Latina, ha estado atravesado por intereses partidistas y hegemónicos, económicos y mercantilistas, producto de la herencia del periodo colonial que se resiste a quedar bajo el influjo de las cadenas opresoras, que nos sumen en atraso e impiden pensar desde una conciencia crítica para construir sociedad.

Misas, (citado por Tarazona, 2004), afirma que:

Hoy, la universidad sigue formando más para el ejercicio profesional que para la investigación científica. Hoy, la universidad sigue reclamando la construcción de una nueva cultura académica que valore la investigación científica, trasfiera el conocimiento como resultado de la investigación y se preocupe por la valoración del conocimiento adquirido. (p.31)

Por ello, es importante repensar la universidad, reflexionar sobre la exigencia de transformarla en Colombia, reflexión ésta que nos sitúa en la obra de Darcy Ribeiro "La Universidad nueva: un proyecto" en la cual afirma que "La Universidad que necesitamos, antes de existir como un hecho en el mundo de las cosas, debe existir como un proyecto, una utopía, en el mundo de las ideas" (Ribeiro, 2006, p.8), es decir, debe ser concebida, pensada desde la realidad oculta a nuestros ojos.

En este sentido, transformar la universidad en Colombia, conlleva pensarla desde un escenario pluridiverso, el desafío está en involucrarla en la vida social, en la construcción de nuevos escenarios educativos, que consoliden una sociedad justa, hacerla participativa, comprometida con el bienestar colectivo, que tome en cuenta la formación para la vida, donde sus estudiantes sean capaces de emitir juicios, producto de las realidades sociales del país, operando como un instrumento transformador.

El artículo presenta cinco secciones: La primera hace una génesis de la universidad en Latinoamérica: La universidad del ayer; la segunda aborda la humanización de la universidad; la tercera se refiere a la universidad vista como eje de cultura y sociedad; la cuarta hace referencia a la universidad promotora y dinamizadora del saber científico como contribución a una sociedad justa; finalmente, se presentan las conclusiones.

\section{Génesis de la universidad en América latina: La universidad del ayer}

Imposible asegurar que, si las universidades desaparecieran, la humanidad sería un caos, perdiendo sentido todo actuar; sin embargo, consideramos que la historia es un elemento importante para entender cómo se concibe en ésta actualidad, cuál es el papel que hoy representa la universidad como Institución, y ante la sociedad. Así, Jacques Lef Goff, (1983,citado por Mollis, 2002) piensa que: "la historia de las universidades es la de las instituciones que contienen saberes para guardar, para distribuir, para descubrir, que se producen, se inventan, se censuran, o simplemente se repiten” (p.328).

En este sentido, la génesis de la universidad en América Latina viene de España, con su proceso de colonización, implementando la educación con carácter religioso, de ahí que las estructuras de las primeras fueran de conventos. Pero, para el siglo XVIII, según Moreno (2005), esto da un 
giro radical, cuando hubo grandes cambios generados en torno a ideas liberales producto de la intolerancia religiosa en las aulas de clase, encargándose entonces los laicos de esta situación.

Las primeras universidades tenían como rol principal la formación de profesionales en Medicina y Derecho, de hecho, el saber profesional caracterizaba el estilo universitario latinoamericano hacia fines del siglo XIX, lo que el historiador alemán Steger, (citado por Mollis, 2002,) describe como la "universidad de los abogados" (p.3); se puede decir incluso que tenían carácter mercantilista, solo personas con recursos económicos podían cursar sus estudios en ellas. Incluso, se puede recordar el discurso de Don Andrés Bello (citado por Moreno 2005, p.72) al inaugurar la Universidad de Chile, donde señalaba que ésta debía ser "un cuerpo eminentemente expansivo y propagador de la cultura del pueblo”.

La universidad, aun cuando desde sus inicios jugó un papel importante en la transmisión de saberes, mantuvo una lucha por la supremacía del poder entre las clases sociales, formando a las élites y a estudiosos de un nivel medio o inferior, pero alejada de la producción y la ciencia. Era criticada por su falta de compromiso con la realidad, con escaso personal docente profesionalizado y carente de una administración eficaz en asuntos universitarios, parafraseando a Brunner \& Catalán (1985, página 58). Así las cosas, Mollis (2002) señala que las universidades encuentran "alterada su identidad que se las asemeja al supermercado, donde el estudiante es cliente, los saberes una mercancía, y el profesor un asalariado enseñante” (p.204).

Hoy en día, la universidad cumple un papel más allá de sus funciones de docencia, extensión e investigación, pero requiere transformarse para transmitir su saber, adaptándose a los cambios sociales, esto exige que ella retome su curso, ser protagonista de una revolución en el mundo de ideas, cuyas características esenciales y su fortaleza interior sean el dinamismo y la flexibilidad permitiéndolo ser ella misma siempre y en todas partes, recuperando su esencia ética, pero sobre todo que sea humanística. Como señala Mollis (2002):
Urge recuperar el significado social, ético y humanista de la calidad educativa. La universidad no sólo produce los conocimientos técnicos y científicos necesarios para el desarrollo del país: sobre todo debe producir saberes necesarios para una construcción democrática, más justa y equitativa; inventar saberes que no estén condicionados por los códigos del lucro; reconstruir su identidad necesaria para nuestras sociedades desprotegidas de individualistas posesivos que niegan el valor de la cultura porque no cotiza en la bolsa de valores. Si la universidad es considerada un elemento del mercado, no hay espacio para la crítica. (p. 212)

De acuerdo con lo anterior, la universidad que se anhela transformar tanto en Colombia, como en América Latina, requiere ser pensada con argumentos sólidos, con una clara idea de que es lo que se quiere, en este sentido De Sousa, José (2002):

Es necesario pensar filosóficamente el cambio y el desarrollo, puesto que sin comprender "por qué” ni con qué propósito iniciamos un proceso de cambio, nos faltarán criterios para saber si lo estamos conduciendo hacia un fin determinado, y nos faltarán argumentos para convencer a otros de que el esfuerzo es necesario y relevante. Observando muchos de los procesos de cambio institucional en marcha en América Latina, nos parece que el cambio, que es solo un medio, se ha transformado en un fin en sí mismo. ¿Por qué deben cambiar las universidades? ¿Para ser más eficientes? ¿Para ser más competitivas? ¿Para mejorar la calidad? ¿Para hacer una reingeniería de su arquitectura organizacional? ¿Para hacer la planificación estratégica? Todo eso se refiere únicamente a los medios. Tal vez la respuesta más sencilla es: para reconstruir su relevancia en la sociedad" porque la sociedad está experimentando un cambio de época. (p. 2)

Esta universidad, afirma Parent (s/f), recibe ahora el impacto de los cambios sociales y técnicos que la obligan a adaptarse. Y, precisamente, aun 
a pesar de las revoluciones a las que ha estado sometida, y los cambios que se han producido, en ese transitar las características esenciales se han venido fortaleciendo con el tiempo, dándole impulso a la energía, así como a la elasticidad que le ayuda a identificarse siempre y en todas partes. Se puede decir que no perderá su función de formar hombres capacitados para la interacción con los demás de una manera creativa conllevando a relaciones nuevas. Así como tampoco obviará el propósito de analizar la realidad científica, en el contexto necesario. De ahí que, todas sus actividades se dirijan al desarrollo de dinamizar procesos investigativos puesto que es la mejor manera de crecer intelectualmente.

Por esto, la universidad de ayer, meramente mercantilista, se debe direccionar a una nueva misión, más humanista, vinculada a la realidad, brindándole al profesional su realización en sus elementos biopsicosociales, que le ayuden a ser más persona, más sensible ante las dificultades y circunstancias del ámbito político, económico y social de las comunidades, que emprenda nuevos roles favorecedores de la cohesión y el bienestar social.

\section{La humanización de la universidad}

Las universidades tienen la titánica tarea de formar individuos para lograr metas a largo o corto plazo, en su vida profesional; pero, la ardua misión de saber cómo enfrentar a una sociedad cada vez más exigente, queda relegada. Son muchos los egresados que tienen que realizar un mayor esfuerzo para poder insertarse en el medio profesional y exitoso de las carreras cursadas. De ahí, cabe preguntarse, iestán los egresados preparados para usar los saberes y convertirlos en un instrumento de servicio a la humanización? o ¿qué está haciendo la universidad para lograr la humanización de sus estudiantes o de los miembros de la comunidad universitaria?

Se sabe que en sus aulas la educación es completamente cognoscitiva y conductual, es decir se aprenden teorías del conocimiento, quizá la aplicación de alguna metodología, pero en cuanto a las técnicas para enfrentar el ejercicio profesional, le corresponde a cada egresado ser creativo ante una sociedad cada vez más envolvente y exigente. $\mathrm{Al}$ respecto, en el contexto del trabajo del voluntariado "La educación que se imparte hoy prepara del peor modo posible para ese cultivo de la acción. La universidad imparte un saber formalista que impele el dogmatismo ideológico o, por reacción, a la ironía estéril” (Aranguren, 2000, p.85).

Se refiere el autor a ese criterio de brindar conocimiento a los estudiantes, saturarlos de teorías, convirtiéndose el docente en un simple trasmisor de éstos, dejando de lado la función de facilitador, de apoyo, capaz de desarrollar ambientes de aprendizaje colaborativo, de plantear problemas en la cotidianidad de la profesión para que se le dé respuesta, porque los profesionales se han formado para lograr hacerse de un capital económico, sin importar los medios empleados para enriquecerse, sin pensar en el otro y en las necesidades colectivas a las cuales debería estar llamado al diseño de estrategias para la satisfacción de las mismas.

En este contexto,

el egresado de una universidad es alguien que ha hecho una promesa pública al recibir su título, y ni su universidad, ni la sociedad en general le han demandado lo que él ha prometido: Poner sus conocimientos al servicio de las necesidades comunes y si no que la sociedad se lo demande. Entonces, si no somos capaces de hacer que nuestros egresados cumplan su palabra, dada la promesa que hicieron frente a los amigos, parientes y autoridades universitarias, antes de recibir su título, ¿verdaderamente los que profesamos en las universidades, estaremos ejerciendo nuestra profesión de manera adecuada? (Robles 2005, s/p)

Debemos cuestionarnos para constatar que se esté formando para la vida, que se estén empleando las estrategias instruccionales y metodológicas adecuadas para la formación de estudiantes. Empero, no es posible crear sociedades alternativas dando la espalda a las capacidades tecnológicas, culturales, empresariales y científicas que hay en un país, es decir, se requiere también la preparación para enfrentar los retos que depara la sociedad y sus 
necesidades locales, que consolide una sociedad justa e igualitaria, comprometida con el bienestar colectivo.

Se trata de humanizar más a nuestras universidades, formando para la vida, instruyendo relaciones interpersonales, en conductas a asumir frente a la sociedad que demanda nuestros servicios profesionales, en concordancia con lo aprendido no solo en conocimiento sino en socialización, trato a las personas, ética profesional y en atención humanitaria a la sociedad en la cual estamos inmersos en esta época de globalización, como política de Estado.

\section{La universidad vista como eje de cultura y sociedad}

Partiendo de una universidad trasmisora de conocimientos, que deja de lado la formación del ser, del conocer, del hacer y del convivir como pilares rectores establecidos por la UNESCO (citado por Delors, 1994, p.91), llama la atención que al excluir estos elementos en este nivel, se produce un divorcio dentro de la formación académica, puesto que en educación básica éstos se encuentran muy presentes, es decir, los niños y jóvenes aprenden a ser, a conocer, a hacer y a convivir juntos en un esquema plural y diverso, empero al llegar a la universidad éstos se centran en el conocer, es decir producir y reproducir elementos cognitivos, pero el estudiante se hace más frío porque no se le prepara en el resto de los principios rectores.

\section{Afirma Delors (1994):}

Para cumplir el conjunto de las misiones que les son propias, la educación debe estructurarse en torno a cuatro aprendizajes fundamentales que en el transcurso de la vida serán para cada persona, en cierto sentido, los pilares del conocimiento: aprender a conocer, es decir, adquirir los instrumentos de la comprensión; aprender a hacer, para poder influir sobre el propio entorno; aprender a vivir juntos, para participar y cooperar con los demás en todas las actividades humanas; por último, aprender a ser, un proceso fundamental que recoge elementos de los tres anteriores. Por supuesto, estas cuatro vías del saber convergen en una sola, ya que hay entre ellas múltiples puntos de contacto, coincidencia e intercambio. (p. 91)

De ahí la importancia, de establecer ejes transversales en la malla curricular en todas las carreras universitarias, direccionadas al de cultura y sociedad, porque así se busca un individuo que se haga copartícipe de los problemas sociales, buscando dar respuesta a las dificultades comunes originadas en las comunidades, desde un punto de vista profesional: el abogado, el ingeniero, el docente, el arquitecto, el sociólogo entre otros, estar sensibilizados para atender a las necesidades propias de la sociedad en la cual se desenvuelve, atendiendo principalmente a la característica de la dignidad humana, comprendiendo que el trabajo en equipo permite logros más eficaces.

\section{Como indica Romero (s/f):}

Este tipo de ejes transversales son de una trascendencia singular porque pretende contribuir con la formación de un profesional que tenga conocimiento de sí mismo, aceptándose tal como es, con sus fortalezas, debilidades, sueños y valores, con aprendizajes y experiencias que le permitan encender su fuego interno, con capacidad para la creatividad e innovación y para superar los paradigmas de cortoplacismo, el conformismo y el individualismo. (p.4)

Es importante señalar, que los aspectos hegemónicos de la cultura y la sociedad deben estar presentes en la formación profesional del ser humano, por ello, es menester conocerse a sí mismo, conocer las costumbres y tradiciones de la sociedad donde vive y poseer conocimiento de las propias necesidades de esta sociedad, a objeto de trabajar para la satisfacción de las mismas desde la profesión adquirida en la universidad.

Para García (1996):

Con las profundas transformaciones que actualmente están ocurriendo en la 
sociedad, hoy más que nunca, la universidad no debe ser ni "militante", ni "enclaustrada”, sino "participante”, como llamó Echavarría (1967) a la universidad deseable, esto es, una institución que tiene el deber y también el privilegio estar en el "flujo" de los cambios y, a la vez, en el "reflujo" de la reflexión sobre lo que implican esos cambios y como deben orientarse a un deber ser que tenga los beneficios deseados en el largo plazo. (p.130)

Por eso se necesita, según Weber (1996), una ciencia que supere la racionalidad instrumental que se proyecte, más allá de lo económico, y supere las barreras mercantilistas, para satisfacer las necesidades de una sociedad más equitativa y humana. Es este enfoque el que De Sousa (citado por Pérez, 2005), sugiere cuando habla de ciencia de la sociedad, "que implica una ciencia con conciencia, con compromiso social, que sitúe los conocimientos al alcance de todos los ciudadanos” (p.3). Una universidad construida por todos y para todos, con sentido crítico y con identidad, que involucre desde los estudiantes, cuerpo docente, hasta la sociedad en general.

De esta manera, debemos actuar y luchar en búsqueda de la transformación necesaria, si queremos una universidad capaz de resolver los múltiples problemas, que se presentan en la sociedad, donde sus estudiantes sean capaces de emitir juicios, productos de las realidades sociales del país, y que estos a su vez operen como un instrumento transformador, según Ribeiro (2006):

Solamente los que estén decididos, a encarnar los intereses de la mayoría de la población y a defender a cualquier costo el desarrollo nacional autónomo, pueden modelar una universidad capaz de actuar como agencia de aceleración evolutiva de la sociedad. Y dentro de la Universidad, sólo el cuerpo estudiantil ofrece suficiente garantía de que no actuará para servir a los objetivos de autoperpetuación de las jerarquías internas y de defensa de los intereses de las viejas clientelas. Por ende, el problema fundamental de la reforma no está en la elaboración técnica de la nueva estructura, sino en la determinación del contenido de poder que marcará el rumbo y el ritmo del proceso de transformación. Este imperativo ineludible señala el cogobierno de las universidades, de los Institutos Centrales, de las de las Facultades y de los Departamentos por sus profesores y estudiantes, como el requisito básico para la edificación de la Universidad necesaria. (p. 170)

En consecuencia, la formación debe ser integral, que abarque su misión profesional, pero aunado a la visión social, a las necesidades generales, a la realidad del pensamiento divergente, de la innovación, por ello el profesional universitario debe encontrarse en capacidad de luchar por sus derechos políticos y sociales, esto se logra no solo formándonos intelectualmente sino también desde el punto de vista de la sensibilización necesaria para conocernos como personas, solo así podemos reconocer al otro en sus requerimientos y derechos.

\section{La universidad promotora y dinamizadora del saber científico como contribución a una sociedad justa y solidaria}

La producción del saber científico y técnico, ya no es solo tarea de la universidad, en tanto academia, por el contrario, desde hace unos años esa actividad se ha ido propagando por toda una sociedad, generando una dinámica distinta, sin la rigurosidad y la articulación que demanda el país, pero dentro del marco de una realidad local o nacional. Sin lugar a dudas la investigación es una de las prioridades que nos compete atender, de ser posible desde el propio seno universitario, para luego proyectarla como política pública, pero que incida en la realidad del país, una investigación que le diga a Colombia hacia donde debe virar su mirada.

Lo que se pretende es la construcción de un nuevo escenario educativo, que consolide una sociedad justa e igualitaria, respondiendo a una visión integradora, y permita abordar problemas fuera de barreras disciplinarias y esquemas pedagógicos tradicionales, desarrolle competencias, conocimientos, habilidades que impacten 
y propicien ambientes abiertos y flexibles de educación. Empero, la universidad en América Latina, posee un defecto, no es más que una copia extraída de las universidades del Hemisferio Norte, aun parecen estar atadas a las cadenas opresoras del proceso colonizador, ello nos lleva a plantear, tal como Varsavsky (2006), que es lo que ha impedido crear una sociedad más justa, solidaria y por sobretodo más humana. Señala:

La universidad enseña hoy una ciencia, una tecnología -física y social-, un concepto del papel del profesionalizar, una actitud hacia la sociedad que son imitación de lo que se hace en el Hemisferio Norte. Este seguidismo cultural refuerza nuestra dependencia económica y dificulta todo intento de reorganizar nuestra sociedad sobre bases más justas y con criterios más humanistas, menos cosificantes. (p. 63)

La investigación, debe entonces ocupar un espacio importante dentro del quehacer universitario, en tanto espacio académico, comprometido con la realidad social. "No es posible, ni necesario tener o aceptar una respuesta prefabricada en otras latitudes, o en las nuestras" (Varsavsky, 2006, p. 64). Es claro que las necesidades de una sociedad difieren de un país, a otro, tal como sucede en Colombia, cuyas realidades si bien son muy similares a otros países, tienen aspectos que la diferencian. En este orden de ideas, aun en Colombia no podemos hablar de una verdadera cultura de investigación, debemos reconocer que en gran parte el trabajo que se adelanta es solitario, que se han venido logrando progresos, con esfuerzo y dedicación, sumado a los docentes que por vocación se han dedicado a la aventura de investigar, sin embargo a pesar de ello, las dificultades económicas y el limitado número de profesionales idóneos y capacitados, impiden la producción científica, la creación de más semilleros de investigación y el desarrollo del país, de la misma manera podría decirse que la investigación que se genera responde todavía en parte a las necesidades y urgencias de otros países.

Bajo estas premisas consideramos que deben generarse líneas estratégicas que permitan desarrollar en las universidades la investigación, con un enfoque definido y dirigido a satisfacer las necesidades locales de la población, solo así podríamos estar convencidos de que la tarea que se emprende va a dejar frutos y va a ser de Colombia un país con grandes potencialidades que le permitan avanzar hacia escenarios de reivindicación del tejido social, y por sobre todo de reconocimiento e identidad propia.

\section{CONCLUSIÓN}

Tomando en consideración todos los señalamientos anteriores, podemos considerar la necesidad perentoria de la transformación universitaria en Colombia, por cuanto ha quedado demostrado el arraigo a la cultura tradicional colonizadora, que se gestó en el momento de la inmersión de la sabiduría española en nuestro territorio y que, indiscutiblemente marcó la pauta de la estructura educativa en todos los niveles, pero que se enraizó en la universitaria.

La universidad del ayer, nos lleva a un entramado cognoscitivo en el cual prevalece la trasmisión de teorías de una forma tradicional e incluso dentro de los enfoques de aprendizaje netamente conductuales, no dando oportunidades al desarrollo de habilidades o destrezas colaborativas, capaces de formar al individuo para encarar las situaciones cotidianas que día a día enfrenta en su profesión. De ahí, que sea necesario un giro revolucionario en las estructuras universitarias, quizá al estilo de Darcy Ribeiro, que resalten el carácter humanista de esta formación. Ciertamente la sociedad requiere de profesiones tradicionales, pero la sobreabundancia de las mismas ha saturado el mercado que la comunidad colombiana puede ofrecer, cerrando las puertas a muchos profesionales que consiguen, en su necesidad, otra forma de vida que a lo mejor no era para la cual se quisieron formar.

En este orden de ideas, es una tarea titánica la que corresponde al Ministerio de Educación, al propio Estado y a las personas que hacemos vida dentro de los recintos universitarios para adecuar las mallas curriculares de las carreras ofrecidas a la satisfacción de las necesidades del pueblo colombiano, insertando como ejes 
transversales los PEI ya que éstos constituyen su carta de presentación a la sociedad, porque le plantea a la formación en la educación superior no sólo posibilidades sino también necesidades de evolución que beneficien la generación de nuevos compromisos sociales, de cara a las variadas pretensiones que el país demanda, buscando el bienestar colectivo y fortaleciendo lazos entre los miembros de la sociedad, a objeto de lograr cambios fructíferos en nuestro territorio, que inclusive puedan conllevar a establecer cultura de paz en el país, al formar profesionales más íntegros y comprometidos con las necesidades sociales.

Finalmente, podemos señalar que al realizar esta transformación a la universidad colombiana, las funciones de academia, investigación y extensión se verían fortalecidas, dando respuesta a los requerimientos de la población colombiana y tomando en consideración al ser humano como único e irrepetible, pero sobre todo con un carácter social por naturaleza, capaz de desarrollar relaciones interpersonales cónsonas con su profesión y de crear empatía con su gente, de desarrollar estrategias creativas que faciliten el desenvolvimiento en una sociedad cada vez más exigente y globalizada, donde la innovación sobresalga, pero sin dejar de resaltar la dignidad de la persona.

\section{REFERENCIAS BIBLIOGRÁFICAS}

Aranguren, L. A. (2000). Vivir es comprometerse. México: IMDOSOC.

Brunner, J. J. \& Catalán, G. (1985). Cinco estudios sobre cultura y sociedad, Editorial Flacso. Santiago de Chile.

Delors, J. (1996). Los cuatro pilares de la educación, en La educación encierra un tesoro. Informe a la UNESCO de la Comisión internacional sobre la educación para el siglo XXI, Madrid, España: Santillana/UNESCO. pp. 91-103.

De Sousa, J. (2002). La Universidad, el Cambio de Época y el Modo Contexto Céntrico de Generación de Conocimiento. CONESUP. Quito. Ecuador. Recuperado de http://es.scribd.com/ doc/46284070/Souza-Modo-Contexto-Centricode-Generacion-de- Conocimiento\#scribd
García, G. C. (1996). Conocimiento, Educación Superior y América Latina. CENDES, UCV. Editorial Nueva Sociedad. Caracas, Venezuela.

González, G. (2006). La Universidad Nueva: Un Proyecto. Fundación Darcy Ribeiro y Fundación Biblioteca Ayacucho, Ministerio del Poder Popular para la Educación Superior Colección Claves de América, No 32 Caracas, Venezuela www.bibliotecayacucho.gob.ve

Misas A., G. (2004). La Educación Superior en Colombia: análisis y estrategias para su desarrollo. Bogotá. Universidad Nacional de Colombia. Bogotá D. C., Colombia.

Mollis, M. (2002a). Un breve diagnóstico de las universidades argentinas: identidades alteradas. En las universidades en América Latina: ¿reformadas o alteradas? La cosmética del poder financiero. CLACSO, Consejo Latinoamericano de Ciencias Sociales. Buenos Aires.

Mollis, M. (2002b). La geopolítica de las reforma de la Educación Superior : el Norte da créditos, el Sur se "acredita". Serie Universidad Contemporánea en colaboración con la Red de investigadores sobre la Educación superior Riseu. Impreso en España. (p. 328)

Moreno, A. (2005). Separata: La Universidad de ayer y hoy. Fundación Konrad AdenauerAtiftung.Universidad Católica Andrés Bello. Vicerrectorado Académico. Caracas. Publicaciones UCAB.

Parent J., J. M. (s/f). La universidad hoy y mañana. Recuperado de http://www. uaemex.mx/plin/. colmena/Colmena37/UAEM175/Parent.html

Pérez Z., L. (2005). Pertinencia social, un reto para la educación superior en el siglo XXI. Centro Universidad Agraria de la Sabana. $\mathrm{V}$ Congreso Internacional virtual de educación. Chile. Recuperado de http://sedici. unlp.edu.ar/bitstream/handle/10915/24506/ Documento_completo.pdf? sequence $=1$

Ribeiro, D. (2006). La universidad nueva: un proyecto. Fundación Biblioteca Ayacucho. Colección claves de América No 32. Caracas-Venezuela.

Robles M. (2005). Cómo humanizar a la universidad. Razón y palabra. No 45. Recuperado de http://www.razonypalabra.org.mx/anteriores/n45/mrobles.html. Consulta 05/08/2015 
Steger, H. A. (1974). Las universidades en el desarrollo social de la América Latina México: Fondo De Cultura Económica

Varsavsky, O. (2006). Hacia una política científica nacional. Monte Ávila Editores Latinoamerica. Caracas-Venezuela. Recuperado de http://es.scribd.com/doc/74932198/ Oscar-Varsavsky-Hacia- una-Politica-CientificaNacional\#scribd

Weber, M. (1996). La política como vocación. En: El político y el científico. Madrid: Alianza Editorial. 